\section{A fenomenologia e as abordagens sistêmicas nos estudos sócio-antropológicos da doença: breve revisão crítica}

\author{
Phenomenology and systemic approaches \\ in socio-anthropological studies of illness: \\ a brief critical review
}

Paulo César Alves 1

\section{Introdução}

This paper aims to identify the ways by which the principal frames of reference in the social sciences explain the structure of social actors' knowledge, practices, values, and behaviors regarding health care. The article begins by examining some of the assumptions underlying studies produced within what are called systemic theories. It then proceeds to discuss phenomenological approaches to the health and illness experience. The goal is to clarify how these approaches focus on experience and depart from prevailing systemic models.

Sick Role; Disease; Health Knowledge, Attitudes, Practice; Social Sciences
Atualmente uma das principais preocupações nos estudos sócio-antropológicos sobre saúde refere-se às pesquisas relacionadas à "experiência da doença”. Emergidas no início da década de 1980 e inicialmente voltadas para a interpretação de doenças crônicas, essas pesquisas passaram a ocupar, nos últimos anos, quase todas as áreas de interesse das ciências sociais em saúde. De uma maneira geral, são trabalhos substancializados pela abordagem fenomenológica-hermenêutica e que expressam uma preocupação em compreender e problematizar como os indivíduos vivenciam uma “experiência de sentir-se mal” e como atribuem significações a esta experiência. Esses estudos apresentam um conjunto de características teórico-metodológicas específicas que contrasta com os principais quadros de referência paradigmáticos que dominaram a história das ciências sociais em saúde.

Com objetivo de identificar algumas das premissas básicas (filosóficas ou epistemológicas) desses estudos, o presente artigo começa por examinar em linhas gerais os pressupostos e tendências das análises sócio-antropológicas tradicionais, que denominamos de "teorias sistêmicas”. De forma mais específica, buscamos inicialmente caracterizar como os principais quadros de referência das "teorias sistêmicas" explicam as concepções, práticas, valores e 
crenças que os atores sociais têm sobre questões relacionadas aos cuidados com a saúde para, em seguida, analisar brevemente em que sentido as pesquisas sobre experiência de doença contrastam com os modelos dominantes.

Desnecessário é dizer que o presente trabalho não pretende ser exaustivo. Serve apenas para mapear algumas questões. Chamamos atenção também para o fato de que utilizamos exclusivamente uma bibliografia estrangeira, principalmente de língua inglesa. Essa escolha não significa dizer que a substancial produção brasileira nessa área tenha uma importância secundária para a discussão sobre a temática em apreço. Apenas procuramos oferecer referências que serviram como textos norteadores da literatura sócio-antropológica internacional e que, de alguma forma, exerceram influências no nosso mundo acadêmico. Por último, observamos que o presente artigo constitui uma atualização de trabalho anteriormente publicado ${ }^{1}$.

\section{O desenvolvimento de teorias sistêmicas}

A primeira grande orientação teórico-metodológica de base sistêmica foi formulada por Parsons 2 . Apesar das críticas feitas a essa teoria, a contribuição parsoniana para o entendimento das determinações sócio-culturais da enfermidade é significativa até os dias atuais. Em termos gerais, a abordagem parsoniana pode ser resumida nos seguintes termos: a saúde constitui um importante pré-requisito funcional para a manutenção de uma dada estrutura social e como a doença se constitui em um desvio da normalidade, é necessário que o doente busque tratamento. Ao assumir a sua condição de doente, isto é, ao adotar o papel de enfermo (sick role), o indivíduo passa a não ser mais considerado como totalmente responsável pelo seu estado, ficando legitimamente isento das suas obrigações sociais normais, desde que procure ajuda competente (dentro dos parâmetros oferecidos pela sua sociedade) e coopere com o tratamento indicado. O papel de paciente articula-se com o do terapeuta e é complementar àquele. Nesse sentido, a abordagem parsoniana parte do princípio de que a medicina se constitui como um sistema, um campo de interação social, e não apenas uma aplicação tecnológica de conhecimentos científicos 3,4,5,6,7,8.

Parsons adotou uma postura "estruturalcoletivista”, alicerçada no princípio da anterioridade ontológica da sociedade. Nessa perspectiva, as normas e valores institucionalizados correspondem a uma realidade objetiva que é internalizada pelos atores. Assim, a ma- neira como os indivíduos compreendem e se engajam ativamente nas situações em que se encontram ao longo de suas vidas é resultado de um sistema coerente e ordenado de idéias, símbolos ou representações. Em outras palavras, o que essa teoria solicita ao pesquisador é que explique o significado das ações humanas por meio da análise das estruturas ou regularidades que estão presentes em dados contextos sociais.

Em que pese as críticas sobre Parsons, é importante salientar que ele abriu um espaço significativo para as análises sociais sobre a enfermidade. Um primeiro aspecto a ser observado é que sua teoria forneceu subsídios para a concepção de "sistema médico", isto é, um modelo conceitual que tem por objetivo explicar o funcionamento de concepções e práticas relacionadas aos cuidados com a saúde. A teoria parsoniana também estabeleceu implicitamente uma separação entre duas concepções de doença: disease e illness. A primeira refere-se usualmente à doença como um processo patológico concebido por um determinado modelo institucionalizado ou profissional da medicina. Enquanto illness, doença diz respeito à percepção subjetiva dos indivíduos e, nesse sentido, envolve questões morais, sociais, psicológicas e físicas 9,10,11. A separação entre esses dois termos fomentou nas analises sobre a medicina ocidental uma oposição idealizada entre os sistemas profissionais e tradicionais no que concerne às concepções de corpo, natureza da doença e papel do terapeuta.

A idéia de "conflito" no sistema de cuidados à saúde foi amplamente desenvolvida na literatura sócio-antropológica. Freidson 12, por exemplo, foi um dos grandes representantes da "teoria do conflito". Pra ele, as diversas definições de doença, principalmente nas sociedades modernas, são determinadas por um sistema pluralístico de valores e princípios sociais. Dessas definições, duas delas são fundamentais: a biomédica, que concebe a doença como desvio de fatores eminentemente morfo-fisiológicos e psicológicos (disease), e a dos pacientes (illness). A primeira é dominante na nossa sociedade devido ao processo social pelo qual os médicos obtiveram um certo monopólio em estabelecer a jurisdição na definição e no tratamento da doença. Pautada pelas referências das ciências biofísicas, a medicina se contrapõe ao "sistema leigo de referência", isto é, um corpo de conhecimentos, crenças e ações que estruturam a percepção leiga do doente. Conforme Freidson 12, é pela análise das estruturas cognitivas que estão subjacentes nos "sistemas leigos de referência” que compreendemos co- 
mo surgem as expectativas sociais envolvidas com a enfermidade e por que elas diferem entre indivíduos pertencentes a um mesmo grupo social.

A literatura sócio-antropológica no campo da saúde foi amplamente marcada, a partir do final da década de 1950, pela produção de inúmeras pesquisas convencionalmente intituladas de illness behavior ("comportamento do doente"), cujo objetivo principal é analisar como os indivíduos e grupos sociais assumem o papel de enfermo de formas diferenciadas 13. Mechanic 14, o criador do termo, juntamente com Kosa \& Robertson 15, entre outros, voltou-se principalmente para analisar os comportamentos, motivações e condutas específicos de indivíduos, isto é, para a explicação de como os sintomas da enfermidade ou do mal-estar são percebidos e avaliados por diferentes tipos de pessoas em diferentes situações sociais. Essa orientação de base "psicossociológica" foi desde cedo contrabalançada por uma abordagem "estrutural-coletivista”, iniciada por Zborowski 16, Kassebaum \& Baumann 17, Zola 18 e Rosenstock 19, a qual enfatiza mais os fatores sócioculturais da doença, chamando atenção para as diferenças de variáveis como classes sociais, faixas etárias e etnias, que atuam nas concepções dos valores e atitudes relacionados à enfermidade.

Embora sigam orientações diferenciadas, ambas as concepções partilham de algumas premissas em comum. Como observam McKinlay 20 e Dingwall 13, os estudos sobre illness behavior estão voltados principalmente para explicar fatores de baixa ou alta utilização dos serviços médicos profissionais e usualmente analisam indivíduos ou grupos sociais que estão ou estiveram sob determinado tratamento e, portanto, que já tomaram a decisão de seguir uma modalidade terapêutica. Assim, tendem a não levar em devida consideração como as decisões foram tomadas e quais os diferentes cursos e possibilidades de ação adotados antes da consulta ou tratamento médico. Além do mais, pressupondo a existência e o poder de normas fixas prescritas, sejam de ordem psicológica ou cultural, subestimam a presença de grupos com aspirações antagônicas na sociedade, a capacidade dos agentes de modificar as regras preestabelecidas ou de redefini-las no decorrer dos processos interativos. Nesse sentido, esses estudos terminam por desencorajar as pesquisas sobre concepções médicas que não estão incluídas nos modelos biomédicos.

Paralelo - e complementando - os pressupostos das teorias sobre o illness behavior, encontramos os estudos intitulados de "represen- tação social". São trabalhos que tratam dos modos pelos quais os indivíduos e grupos sociais nomeiam e definem conjuntamente os diferentes aspectos da realidade, interpretam seus contextos e tomam decisões. A "teoria da representação” pretende trazer concomitantemente para a análise a marca do sujeito que se reporta a um objeto, as estruturas mais amplas de pensamento (ideológicos ou culturais) e as condições sociais efetivas dos indivíduos e grupos. Para os seus teóricos, as representações formam "sistemas de interpretação" que regem nossas relações com o mundo e com os outros, orientam e organizam as condutas e as comunicações sociais. Em outras palavras, as representações são "sistemas cognitivos" resultantes da interiorização de modelos de conduta e pensamento, socialmente inculcados ou transmitidos por processos sociais.

O termo representação tem uma longa, diversificada e ambígua história. O uso que a psicologia social faz desse termo não é consensual. Representação ora é equiparada com a percepção, com a lembrança, com a imaginação ou até mesmo com a alucinação. Nas ciências sociais, Durkheim foi o primeiro a fixar os contornos desse conceito ao identificar as idealizações coletivas como produções mentais e a estabelecer um isomorfismo entre elas e as instituições. Para uma análise durkheimiana sobre a doença ver Taylor \& Ashworth 21. Fundamentado em Durkheim, Serge Moscovici retomou e expandiu esse conceito, situando-o na interface do psicológico e do social. Para esse autor, a representação social tem com o seu objeto uma relação de simbolização e de interpretação (conferindo-lhe significações) que é resultado de uma construção e uma expressão do sujeito. É uma forma de saber que se refere à experiência a partir da qual ele é produzido, aos contextos e condições em que ele o é. A representação preenche, portanto, certas funções na manutenção da identidade social e do equilíbrio sociocognitivo a ela ligados.

Atualmente um conjunto significativo de pesquisadores no campo da saúde desenvolvem pesquisas sob a ótica das "representações sociais”, como Denise Jodelet, Rory Williams, Claudine Herzlich, Janine Pierret e François Laplantine, para citar apenas alguns. Em termos gerais, essa teoria fundamenta-se no pressuposto de que, ao descrever e explicar seus sofrimentos, os indivíduos apóiam-se em conceitos, estruturas de referências e "visões de mundo” interiorizadas conforme os grupos sócioculturais a que pertençam. Em outras palavras, as representações são formas de conhecimento ou sistemas de interpretação, socialmente 
elaboradas e partilhadas, que orientam e organizam as condutas e as comunicações sociais.

Em resumo, as "teorias sistêmicas" partem de um axioma central: as propriedades coativas ("normativas") da organização social são "introjetadas" nos indivíduos. Mais especificamente, a matriz fulcral dessas teorias é a de que o ator é definido pela interiorização do social e a ação é simplesmente a realização das normas de um conjunto social previamente estabelecido. A partir desse teorema, as análises desenvolvidas podem se concentrar ou sobre a integração de elementos motivadores, culturais ou simbólicos, unidos em uma determinada espécie de sistema ordenado (a ênfase, portanto, recai no "problema da ordem" e do "controle" nas interações sociais como nas abordagens influenciadas pelas teorias durkheimiana ou parsoniana) ou sobre os conflitos, poder e mudanças sociais nos processos interativos (concepção defendida, entre outras, pela orientação marxista de cunho estrutural). Qualquer que seja o ângulo de análise, contudo, ambas afirmam tanto o primado transcendente e ontológico da sociedade (como "prévio" aos indivíduos) quanto a existência de "leis", "modelos”, "códigos” ou "estruturas” regidas por princípios intrínsecos a qualquer forma "ordenada" da vida social. Essa tese é problemática, entre outros aspectos, porque estabelece como ponto de partida analítico uma dicotomia - ou oposição - entre o "indivíduo" (visto como "ator abstrato") e a "sociedade" (pensada como um "modelo" ou um "padrão normativo comunitário"). Assim, a "sociedade" é concebida como um universo substantivo e "pré-dado" de objetos e idéias. Nessa perspectiva, há uma forte tendência em minimizar o ator como um agente criativo, inovador, reflexivo e que às vezes procura romper com os esquemas nos quais a conduta humana não se diferencia. Por outro lado, uma concepção dicotômica entre indivíduo e sociedade também pressupõe a existência apriorística de um "sujeito" sobre o qual recaem os componentes reguladores de um dado sistema social. Assim, as "teorias sistêmicas" ao postular a existência prévia do indivíduo - o "sujeito do social" - terminam por admitir determinadas dimensões "a-históricas" (ou mesmo, "anti-históricas") do ser humano.

\section{Experiência da enfermidade: a emergência de um conceito}

Os estudos sobre experiência da doença tomam como ponto de partida analítico o pressuposto de que o social é originalmente toda a situação vivida pelos atores sociais em contextos culturais e históricos concretos. O "mundo da vida" ("Lebenswelt") é o horizonte de referência paradigmático por meio do qual os indivíduos e grupos sociais interagem entre si, definem ações previstas de sentido e realizam qualquer empreendimento cognitivo, como as "representações". As experiências originalmente fundadas dentro do mundo da vida constituem a base sobre a qual se erguem todas as demais "realidades" humanas, como o "sonho", a arte, os sistemas simbólicos e o fazer científico, por exemplo. É, portanto, o mundo das atividades práticas que possui desde um primeiro momento significações humanas.

Os primeiros grandes estudos sobre experiência da enfermidade começaram a surgir no início da década de 1980. Byron Good e Arthur Kleinman, ambos professores da Universidade de Harvard, foram os principais pesquisadores que inicialmente deram um caráter mais sistematizado a essas pesquisas. Fundamentado teoricamente pelos trabalhos de Berger \& Luckmann 22 e por Geertz 23, Good 24,25 e Kleinman 26,27 conceberam o conceito de "modelos explicativos da doença" como um ponto de partida das suas pesquisas. Esses modelos são definidos como noções, conjunto de proposições ou generalizações, explícitas ou tácitas, que são empregadas por todos aqueles indivíduos engajados em um processo terapêutico. Eles servem para determinar o que é considerado como "evidência clínica relevante" e como esta é organizada e interpretada em tratamentos específicos. Estruturalmente, os modelos diferem entre si na resposta que oferecem à etiologia, ao tempo e modo do aparecimento de sintomas, ao curso e gravidade da doença e do tratamento. Os modelos explicativos referem-se, portanto, a conhecimentos e valores que são construídos socialmente pelos diferentes "sistemas de cuidados à saúde" (health care systems).

Como regra, os teóricos dos "modelos explicativos" observam que os sistemas de cuidado à saúde são compartimentalizados, divididos em subsetores. Para Kleinman 26,28,29, a estrutura desses sistemas é composta por três grandes arenas: profissional, "folk" e o popular. Embora sejam entendidos em relação um com os outros, esses três setores guardam suas próprias especificidades com relação às crenças, papéis, expectativas, avaliações e concepções médicas ("realidades clínicas"). A primeira arena diz respeito às práticas e saberes que são constituídas por um conhecimento científico ou por uma tradição específica de cuidados à saúde como a biomedicina, a homeopatia, a medicina chinesa, a Yunani e Ayurvedica. O 
"folk" refere-se às atividades de tratamento e cura desenvolvidas por grupos religiosos, rezadores, curandeirismo etc. O popular é o setor composto pelos "leigos" e constitui o mais importante dessas três arenas, pois nela se concentra a maioria dos nossos saberes e práticas cotidianas que estão relacionadas ao fenômeno saúde-doença. São pelos pressupostos desse setor que diferentes indivíduos e grupos sociais constroem concepções de tratamento e cura; ingressam em um itinerário terapêutico; assimilam, avaliam, julgam os conhecimentos e práticas provenientes dos outros setores. A teoria dos "modelos explicativos" (MEs) nos permite estabelecer, portanto, quadros analíticos comparativos entre diferentes culturas e sistemas de tratamento (ver Kleinman 28,29 e Young ${ }^{30}$ ).

Dentro dessa perspectiva, alguns pesquisadores argumentam, a exemplo de Harrel 31, que em contextos pluralísticos, os subsistemas médicos tendem a se tornar ligados a doenças específicas, de tal forma que os padrões de procura de auxílio (pathways of resort) podem ser delineados por diferentes tipos de situação de enfermidade. Afirmam, portanto, que certos tipos de práticas são considerados socialmente como os mais adequados para explicar certos tipos de aflição. Para Kleinmam 26, os diversos subsetores terapêuticos não instituem fronteiras definidas entre si, podendo ser interconectados de diversas formas. Conforme esse antropólogo, os relacionamentos sociais estabelecidos por indivíduos e grupos destinados ao cuidado com a saúde - como, por exemplo, nas relações entre paciente-família e paciente-terapeuta - devem ser estudados e comparados como transações entre diferentes modelos explicativos. O argumento de Kleinman chama atenção para o fato de que não podemos considerar as concepções populares sobre a doença como pertencentes a um "modelo unitário" mas, pelo contrário, como resultado das experiências pessoais, das combinações e interações que os atores e grupos sociais desenvolvem em diferentes contextos.

A teoria dos "modelos explicativos" constituiu uma reviravolta significativa nos estudos sócio-antropológicos da saúde e uma importante porta de entrada à análise fenomenológica. Cabe notar, porém, que os teóricos dos MEs tendem muitas vezes a explicar os conhecimentos e crenças que os indivíduos têm sobre questões de saúde e doença em termos de estruturas cognitivas. Assim, terminam por priorizar as representações e não propriamente as experiências que os atores sociais têm sobre o mundo em que vivem. Contudo, vale salientar aspectos diferenciadores na teoria dos "modelos explicativos”. Resumidamente, essa concepção: (a) critica as formulações "hipersocializadas” da ação, isso é, aquelas que ao postular uma programação total ou puramente estratégica da ação terminam por fazer do indivíduo, no dizer de Garfinkel, um "idiota cultural”; (b) sublinha que existe na experiência alguma coisa de inacabado e de opaco porque não há adequação absoluta da subjetividade do ator e da objetividade do sistema; (c) advoga que a experiência não é pura questão individual, pois é socialmente construída (ela só existe verdadeiramente, aos olhos do indivíduo, na medida em que é reconhecida por outros, eventualmente partilhada e confirmada por outros); (d) argumenta que ação é definida pela natureza das relações sociais (ação é uma orientação subjetiva e, simultaneamente, uma relação); (e) sustenta uma tipologia da "ação múltipla", isso é, a de que não existe um sistema e uma lógica da ação, mas uma pluralidade não hierárquica e uma "tensão" entre lógicas diferentes; (f) afirma que a tarefa do pesquisador não é analisar apenas as representações do ator social mas, principalmente, os seus sentimentos e a relação que ele constrói com ele mesmo e com os outros.

\section{A experiência da doença e as ciências sociais de base fenomenológica: fundamentos teóricos}

O conceito básico nos trabalhos desenvolvidos por cientistas sociais que utilizam os quadros de referências de base fenomenológica e hermenêutica - como a etnometodologia, a análise conversacional e a sociologia existencial - é o de experiência.

Embora o termo "experiência" seja amplamente empregado pelos cientistas, há pouca concordância e muita polêmica sobre o seu significado. Trata-se de uma noção que apresenta vários sentidos e, muitas vezes, é utilizado de forma demasiadamente vaga nas análises. Usualmente é concebida como ensinamento adquirido com a prática (quando se fala, por exemplo, em "experiência de vida") ou como a confirmação dos juízos sobre uma realidade por meio de uma verificação sensível (as chamadas "experiências científicas") ou então para indicar um fato de alguém suportar ou de sofrer algo ("experiência de uma dor"). Para o movimento fenomenológico, experiência é a forma original pela qual os sujeitos concretos vivenciam o seu mundo. Em outras palavras, experiência diz respeito ao modo de ser do su- 
jeito no mundo. É o meio pelo qual o mundo se coloca face a nós e dentro de nós e, como tal, está sempre localizada no tempo e no espaço. Nesse aspecto, as pesquisas desenvolvidas por esse referencial insistem na reabilitação do sensível, de que as tradições da filosofia e das ciências sociais sempre desconfiaram.

O ponto de partida é "voltar às próprias coisas”, segundo o programa husserliano de transcender as representações espontâneas do senso comum. O primeiro passo do método fenomenológico é reconhecer a prioridade da prática, da esfera do fazer e agir, sobre o pensamento e a reflexão. A experiência não se resume à capacidade humana de representar o mundo por meio de processos cognitivos, pois guarda em si mesma "significações" ou "unidades ideais significativas". Para a fenomenologia, a operação primordial de significação é que o expresso não existe separadamente da expressão e, como tal, é "encarnada" (embodiment), ou seja, é um fenômeno sensível ao corpo (e não uma mera questão de "subjetividade"). Nesse aspecto, o método fenomenológico está voltado para apreender as significações na medida em que são simplesmente dadas e tal como são dadas pelas nossas experiências. Ou seja, preocupa-se com o solo originário do sentido, com o implícito que prepara a explicitação. Por conseguinte, as ciências sociais de base fenomenológica propõem descrever o "que se passa" efetivamente do ponto de vista daqueles que vivem uma dada situação concreta e como, por meio desse processo, os indivíduos e grupos sociais concebem reflexivamente ou representam o seu mundo. Contudo, é necessário observar que nenhuma análise é definitiva. O real contém uma infinidade de "significações" que é necessário trazer à luz e os esquemas de inteligibilidade possíveis estão em constante transformação.

Três aspectos são fundamentais para a análise fenomenológica: corpo, compreensão e intersubjetividade

Colocar o acento sobre o domínio da experiência pressupõe, como já observado, resgatar a mediação do corpo enquanto fundamento de nossa inserção prática no mundo. Para a fenomenologia, o corpo é o entrelaçamento entre natureza e cultura e desempenha o papel fundamental de colocar-nos em contato com o mundo. Tal postulado nos leva a novas questões sobre a experiência da doença. Por exemplo, como observa Csordas 32,33, a doença nos conduz a problematizar o processo mesmo quando a vivência do sentir-se mal se constitui e ganha expressão.
Na vontade de explicitar constantemente as camadas de sentido mais originárias, a fenomenologia torna-se uma hermenêutica e, como tal, alia-se ao quadro de referência da "compreensão" ("Verstehen"). Atribui, contudo, um novo sentido para a hermenêutica: não é simplesmente um método para compreender as ações humanas (como pressupunha Dilthey) mas, prioritariamente, a base sobre a qual se fundamenta toda compreensão. A compreensão não é uma faculdade humana entre outras, é o modo essencial que o homem tem de existir no mundo. Ela está subjacente a tudo, pois é sempre a posição a partir da qual vemos tudo aquilo que vemos. Assim, compreender é experienciar algo. Para um estudo sobre saúde com base na hermenêutica ver Gadamer 34.

O terceiro aspecto fundamental da análise fenomenológica diz respeito à idéia de intersubjetividade. Para essa perspectiva, o social não é soma de subjetividades e tampouco uma realidade objetiva (como "estrutura simbólica" ou integração entre sistemas), mas modos de coexistência entre indivíduos, isso é, processos de interação com os outros desenvolvidos na esfera da vida cotidiana. O encontro com o outro não é, portanto, uma realidade contingente da ação humana, mas o próprio campo do qual torna-se possível os nossos esforços para intervir na realidade. A intersubjetividade refere-se justamente a essa "compreensão mútua" que preexiste nas relações entre os diversos "eus", isso é, o mundo compartilhado por todos nós.

Tomando como ponto de partida a idéia de intersubjetividade, as ciências sociais de base fenomenológica compreendem a doença como um processo de rompimento com os pressupostos da vida cotidiana, daí as dúvidas, incertezas e vacilações que marcam muitas das experiências cotidianas de adoecer e lidar socialmente com a enfermidade 35,36 . Só nesses termos podemos entender por que a doença ou sofrimento, ao romper com o caráter dado ou pressuposto de esferas da vida cotidiana, coloca em questão aspectos da conduta dos indivíduos e grupos sociais. Eis por que a preocupação que alguns pesquisadores têm em analisar a doença como disrupção através de "história de vida" 37,38,39,40,41,42,43,44,45.

Em síntese, partindo da premissa de que a intersubjetividade é o solo sobre qual são elaboradas as diversas interpretações do mundo da vida cotidiana, para a fenomenologia-hermenêutica a doença, antes de ser um fenômeno de disease ou de illness, é originalmente sickness. Isso quer dizer que a doença é um fenômeno que diz respeito a um conjunto de elementos sócio-culturais que estão interligados 
entre si. Sickness refere-se ao "mundo da doença", isso é, a um horizonte de significados, condutas e instituições associadas à enfermidade ou ao sofrimento.

\section{Conclusão}

O objetivo geral do presente artigo foi tão somente explicitar - e contrastar - alguns dos pressupostos (filosóficos ou epistemológicos) que estão subjacentes em grande parte dos trabalhos sócio-antropológicos da saúde. Longe de ser exaustivo, apresentamos brevemente duas grandes linhas de análise sobre como esses estudos interpretam as formas pelas quais os atores sociais pensam e atuam em questões relacionadas aos cuidados com a doença ou sofrimento. A primeira diz respeito às premissas de caráter "sistêmico"; a segunda, de ordem "fenomenológica-hermenêutia".

Para concluir, chamamos atenção para o fato de que as ciências sociais de base fenomenológica-hermenêutica apresentam desafios metodológicos significativos. Não há espaço aqui para discutir esses desafios (ver, para isso, os trabalhos de Cicourel 46, Ten Have 47, Holstein \& Gubrium 48 e Moustakaf 49 ). Observamos apenas que um pressuposto fundamental da metodologia proposta pelas ciências sociais de base fenomenológica é a de que o pesquisador desenvolva uma cuidadosa atenção para os processos interativos e discursivos, particularmente para as situações e contextos do objeto de investigação. Partindo-se da premissa de que as "realidades objetivas" são resultados desses processos, a etnometodologia, a análise conversacional e a sociologia existencial sustentam que significação nunca é completamente predeterminada; ela está sempre articulada em contextos interativos específicos. Nesse aspecto, as narrativas, relatos e descrições elaborados pelos indivíduos não se resumem apenas ao que é dito nesses discursos sobre o mundo social mas, principalmente, são elementos constitutivos desse mundo. Como observam Holstein \& Gubrium 48 , essas abordagens não buscam informações generalizadas sobre interação e discurso por meio de entrevistas estruturadas ou questionários. Para compreender como são produzidas as ordens sociais em determinados contextos, as pesquisas recorrem principalmente a conversas e diversas modalidades de discursos, entre eles cartas, depoimentos e até mesmo textos literários.

\section{Resumo}

O presente artigo tem por objetivo identificar, em linhas gerais, como os principais quadros de referência no campo das ciências sociais explicam as concepções, as práticas, as vivências, os valores e atitudes que os atores sociais têm sobre questões relacionadas aos cuidados com a saúde. Examina inicialmente alguns dos pressupostos aos estudos nessa área orientados por "teorias sistêmicas", para em seguida explicitar em que sentido os estudos de base fenomenológica, ao enfatizar a temática da experiência da doença, contrastam com os modelos dominantes.

Papel do Doente; Doença; Conhecimentos, Atitudes e Prática em Saúde; Ciências Sociais

\section{Referências}

1. Alves PC. A experiência da enfermidade: considerações teóricas. Cad Saúde Pública 1993; 9:263-71.

2. Parsons T. The social system. Glencoe: Free Press; 1951.

3. Gerhardt U. The parsonian paradigm and the identity of medical sociology. Sociol Rev 1979; 27:229-51.

4. Gerhardt U. Parsons, role theory, and health interaction. In: Scambler G, editor. Sociological theory and medical sociology. London: Tavistock Publications; 1987. p. 110-33.

5. Gerhardt U. Ideas about illness. An intellectual and political history of medical sociology. Houndmills/London: MacMillan Education; 1989.

6. Erikson K. Patient role and uncertainty: a dilemma of the mentally ill. Psychiatry 1957; 20:263-74.

7. Petroni FA. Significant others and illness behavior: a much neglected sic role contingency. Sociol Q 1969; 10:32-41. 
8. Denzin N, Spitzer S. Paths to the mental hospital and staff predictions of patient role behavior. J Health Hum Behav 1966; 7:265-71.

9. Fabrega $H$. The need for an ethnomedical science. Science 1975; 189:969-75.

10. Eisenberg L. Disease and illness: distinctions beteween professional and popular ideas of sickness. Cult Med Psychiatry 1977; 1:9-23.

11. Mitchell WE. Changing others: the anthropological study of therapeutic systems. Med Anthropol Newsl 1977; 8:15-9.

12. Freidson E. Profession of medicine. New York: Dodd and Mead; 1970.

13. Dingwall R. Aspects of illness. London: Martin Robertson; 1976.

14. Mechanic D. Illness and social disability: some problems of analysis. Pac Sociol Rev 1959; 2:37-41.

15. Kosa J, Robertson LS. The social aspects of health and illness. In: Kosa J, Zola T, editors. Poverty and health. Cambridge: Harvard University Press; 1969. p. 40-79.

16. Zborowki M. Cultural components in responses to pain. J Soc Issues 1952; 8:16-30.

17. Kassebaum GG, Baumann BO. Dimensions of the sick role in chronic illness. J Health Hum Behav 1965; 6:16-27.

18. Zola IK. Culture and symptoms. An analysis of patients' presenting complaints. Am Sociol Rev 1966; 31:615-30.

19. Rosenstock IM. Why people use health services. Milbank Mem Fund Q 1966; 44:94-127.

20. McKinley JB. Some approaches and problems in the study of the use of services: an overview. J Health Soc Behav 1972; 13:115-52.

21. Taylor S, Ashworth C. Durkheim and social realism: an approach to health and illness. In: Scambler G, editor. Sociological theory and medical sociology. London: Tavistock Publications; 1987. p. 37-58.

22. Berger PL, Luckmann T. The social construction of reality. New York: Doubleday; 1967.

23. Geertz C. The interpretation of cultures. New York: Basic Books; 1973.

24. Good B. The heart of what's the matter: the semantics of illness in Iran. Cult Med Psychiatry 1977; 1:25-58.

25. Good B. Medicine, rationality, and experience. Cambridge: Cambridge University Press; 1994.

26. Kleinman A. Patients and healers in the context of culture. An exploration of the borderland between anthropology, medicine and psychiatry. Berkeley: University of California Press; 1980.

27. Kleinman A. The illness narratives: suffering, healing, and the human condition. New York: Basic Books; 1988.

28. Kleinman A. Toward a comparative study of medical systems: an integrad approach to the study of the relationship of medicine to cultures. Sci Med Man 1973; 1:55-65.
29. Kleinman A. Concepts and a model for the comparison of medical systems as cultural systems. Soc Sci Med 1978; 12:85-93.

30. Young A. Internalizing and externalizing medical belief systems: an ethiopian example. Soc Sci Med 1976; 10:147-56.

31. Harrel S. Pluralism, performance and meaning in Taiwanese healing: a case study. Cult Med Psychiatry $1991 ; 15: 45-68$.

32. Csordas T. Embodiment as a paradigm for medical anthropology. Ethos 1990; 18:5-47.

33. Csordas T. Somatic modes of attention. Cult Anthropol 1993; 8:135-56.

34. Gadamer HG. El estado oculto de la salud. Barcelona: Editorial Gedisa; 1996.

35. Atkinson P. The clinical experience: the construction and reconstruction of medical reality. London: Gower; 1981.

36. Lynch M. Accommodation practices: vernacular treatments of madness. Soc Probl 1983; 31:152-64.

37. Bury M. Chronic illness as biographical disruption. Sociol Health Illn 1982; 4:167-82.

38. Williams $\mathrm{G}$. The genesis of chronic illness: narrative reconstruction. Sociol Health Illn 1984: 6:175200 .

39. Frank G. On embodiment: a case study of congenital limb deficiency in American culture. Cult Med Psychiatry 1986; 10:189-219.

40. Kaufman S. Illness biography and the interpretation of self following a stroke. J Aging Stud 1988: 8:217-27.

41. Garro L. Chronic illness and the construction of narratves. In: Good MJ-D, Brodwin P, Good B, Kleinman A, editors. Pain as human experience: an anthropological perspective. Berkeley/Los Angeles: University of California Press; 1992. p. 100-37.

42. Becker G. Metaphors in disrupted lives: infertility and cultural construction of continuity. Med Anthropol Q 1994; 8:383-410.

43. Becker G. Disrupted lives: how people create meaning in a chaotic world. Berkeley/Los Angeles: University of California Press; 1997.

44. Mattingly C, Garro L. Introduction: narrative representations of illness and healing. Soc Sci Med 1994; 38:771-4.

45. Mattingly C, Garro L. Narrative and the cultural construction of illness and healing. Berkeley/Los Angeles: University of California Press; 2000.

46. Cicourel AV. Method and measurement in sociology. New York: Free Press; 1964.

47. ten Have P. Methodological issues in conversation analysis. Bull Methodol Sociol 1990; 27:23-51.

48. Holstein JA, Gubrium JF. Phenomenology, ethnomethodology, and interpretative practice. In: Denzin NK, Lincoln YS, editors. Handbook of qualitative research. London: Sage Publication; 1994. p. 262-72.

49. Moustakaf K. Phenomenological research methods. London: Sage; 1994.

Recebido em 26/Out/2005

Versão final reapresentada em 20/Mar/2006

Aprovado em 31/Mar/2006 